\title{
Filigrane
}

Écoutes psychanalytiques

\section{Le merveilleux surgissement des rencontres inattendues. Recension du collectif Des psychanalystes en séance}

\section{Stephany Squires}

Volume 27, numéro 1, 2018

URI : https://id.erudit.org/iderudit/1055605ar

DOI : https://doi.org/10.7202/1055605ar

Aller au sommaire du numéro

Éditeur(s)

Revue Santé mentale au Québec

ISSN

1192-1412 (imprimé)

1911-4656 (numérique)

Découvrir la revue

Citer ce compte rendu

Squires, S. (2018). Compte rendu de [Le merveilleux surgissement des rencontres inattendues. Recension du collectif Des psychanalystes en séance]. Filigrane, 27(1), 101-108. https://doi.org/10.7202/1055605ar d'utilisation que vous pouvez consulter en ligne. 


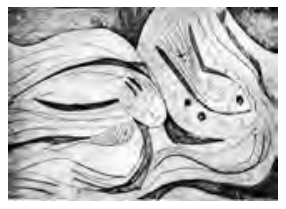

\title{
Le merveilleux surgissement des rencontres inattendues. Recension du collectif Des psychanalystes en séance
}

\author{
Stephany Squires
}

\begin{abstract}
Quand en psychiatrie s'impose un homme comportemental déterminé par son patrimoine neurogénétique, tous les savoirs de l'homme du conflit, de l'homme psychique, du rêve, de la poésie, c'est-à-dire de l'homme freudien, sont exclus du paysage institutionnel. (Roland Gori, 2010)
\end{abstract}

et article se veut d'abord un compte rendu du lexique intitulé Des psychanalystes en séance. Glossaire clinique de psychanalyse contemporaine (2016) pour ensuite proposer une réflexion critique sur le sujet de la transmission de la psychanalyse au sein de nos institutions universitaires québécoises. Il s'adresse à ces collègues qui cherchent, en des mots et en des lieux, les possibilités réflexives situées entre l'héritage et l'à-venir psychanalytiques, là où les porte-voix présentés dans ce glossaire permettent d'ouvrir des horizons, de décloisonner les murs privés que sont ceux de la clinique.

Ce lexique propose une rencontre où cinquante-sept analystes - d'ailleurs tous présentés brièvement dans l'appendice - unissent leurs voix et nous invitent à voir et à entendre ce que l'intime de l'analyse peut révéler derrière l'édifice métapsychologique. Ces fragments racontent davantage qu'ils n'enseignent: un glossaire structuré comme un dictionnaire, mais plus proche du journal clinique si l'on veut. En effet, il est possible d'ouvrir ce glossaire tel un dictionnaire où nous allons chercher une compréhension théorico-clinique sous une rubrique bien définie. Pour ma part, je l'ai lu comme on plonge dans un recueil de nouvelles où les histoires s'enchaînent sous le signe du singulier, du raconter, de l'historicité. En effet, ces «petites» théories prennent corps sous la finesse de cas cliniques illustrés et nous captons bien que "chaque séance d'analyse est ainsi faite, unique mais répétée » 
(Danon-Boileau et Tamet, 2016, p. 57). Comme l'énoncent Laurent DanonBoileau et Jean-Yves Tamet dans l'introduction, ce sont certes des analystes d'horizons parfois très différents avec par exemple des thèmes passant de «La capacité négative et capacité de rêverie» (2016, p. 130) à "L’objet partiel ou objet (a)» (2016, p. 357), mais cela n'enlève rien à la cohérence qui perdure tout au long de cet ouvrage, si ce n'est la rigueur réflexive qui demeure au service de la pensée théorico-clinique. Enfin, à l'image d'une courtepointe dont la beauté ne réside aucunement dans l'uniformité, mais plutôt dans le tissage délicat des différentes couleurs et textures qu'offre son assemblage unique, la lecture de ce glossaire démontre qu'il est possible de créer des ponts entre différentes théories sans compromis par rapport à la sensibilité clinique, et ce, bien loin d'une orthodoxie rigide.

D'un point de vue logistique, le tout est brillamment orchestré dans un maniement sensible des pensées et des penseurs, sous des sections qui aident à garder le fil entre les deux grandes catégories proposées qui sont: l'espace de la séance et l'espace de la psyché. Cela va sans dire, la richesse des bibliographies retrouvées sous chaque thème est un point fort de ce glossaire, voire une invitation à sortir de ses zones de confort théorique. Toujours dans sa logique structurelle, l'aspect pratique et intuitif offre une facilité dans la recherche de thèmes, d'idées et même d'auteurs. Par ailleurs, une mention spéciale doit être faite pour l'index grâce auquel chercher un thème s'effectue en un tour de main!

Comme l'indique le titre de ce glossaire, les thèmes choisis épousent les lignes cliniques dites contemporaines. Or, ce survol d'autant de penseurs analytiques actuels permet de s'ouvrir aux remaniements et aux avancées effectuées jusqu'à ce jour, sans pourtant nier l'héritage fondamental transmis au fil du temps en commençant par Sigmund Freud lui-même. À titre d'exemple, Mireille Fognini fait des liens entre le concept de traces mnésiques de S. Freud et celui du pictogramme de l'originaire de Piera Aulagnier (Danon-Boileau et Tamet, 2016, p. 75). Or, cela n'empêche pas cette psychanalyste de faire valoir les avancées depuis Freud, notamment sous la rubrique «La polyphonie du rêve» (Danon-Boileau et Tamet, 2016, p. 92). En effet, elle souligne que la théorie autour du rêve a d'abord été pensée par Freud dans un apport individuel/intrapsychique, mais qu'aujourd'hui, avec les apports de René Kaës entre autres, le rêve devient aussi «collectif» dans le vaste champ transféro-contre-transférentiel effleuré. N'est-ce pas un travail, non suffisant, mais nécessaire, à l'avancée réflexive de la psychanalyse que d'être capable de reconnaître les apports freudiens, et de continuer 
à parcourir les sens premiers pour ensuite mettre en lumière ce qui était resté dans l'ombre par des éclairages nouveaux? Toujours dans cette même section, nous sommes témoins de la correspondance entre M. Fognini et R. Kaës dans laquelle nous pouvons sentir un mouvement réflexif partagé. Cela n'est pas sans rappeler l'importante place de la correspondance chez Freud.

De plus, il me semble que l'exercice d'élaborer, en si peu de mots, autour de concepts aussi complexes qu'intimes, tel que le font Alice Bauer-Torrente et Joseph Torrente par exemple entre la pensée paradoxale et la régrédience (Danon-Boileau et Tamet, 2016, 148-157) montre l'exigence et la sensibilité déployées dans ce glossaire. Au passage, Claire Tremoulet souligne, à la fin de sa section consacrée à «L'analogie», les remaniements nécessaires quant aux mouvements et enrichissements auxquels invite l'expérience de l'analyse elle-même, et elle écrit:

C'est en ce point précis que réside l'intérêt du travail de Jean-Claude Rolland, quand il transforme un mot de la langue d'usage courant en un mot qui vient enrichir l'expérience de la cure, et la nôtre par cet apport original. N'est-ce pas ainsi que progresse la théorie? (Danon-Boileau et Tamet, 2016, p. 87).

La diversité des sources utilisées par les cliniciens et cliniciennes de ce recueil m'a fait voyager. Ils nous sortent de nos cloisons théoriques, institutionnelles et temporelles, cloisons que l'on pourrait rattacher à des effets de modes ou d'appartenances qui parfois nous écartent d'emblée, plutôt que de nous faire garder cette même curiosité envers la théorie que l'on se voudrait avoir envers les patients. Au cours de cette traversée presque littéraire, je me mis à penser, mais pourquoi est-ce la première fois que j'entends parler de ce concept, de cet auteur ou encore de cette façon? Peut-être que ma plus grande question, finalement, revient à celle-ci: quelle place doit-il y avoir, dans nos universités, pour l'enseignement de la psychanalyse?

Comme annoncé dans l'introduction de cet article, cette deuxième section témoignera de la manière dont la lecture de ce glossaire m'a ouvert le chemin vers l'épineux sujet qu'est celui de la transmission psychanalytique dans nos universités québécoises. Je terminerai donc sous les lumières réflexives qu'offrent deux psychanalystes québécois, de deux générations différentes, dont les regards et les discours se dialectisent suffisamment bien pour en faire ressortir les grandes lignes. Il s'agit de feu André Lussier et 
de Nicolas Lévesque, chacun m'étant apparu dans les dédales des mots, des textes et des traces laissés à travers ces détours et contours qu'a fait résonner en moi ce glossaire.

Je proposerais que l'association libre, la liberté d'association et la transmission (formation et enseignement) entretiennent des liens jusqu'à ce jour peu relevés. Afin d'illustrer cette transposition du procédé de l'association libre propre à l'analyse dans le cadre universitaire, je vais décortiquer les deux termes de cet énoncé. D’abord, dans le Vocabulaire de psychanalyse de Laplanche et Pontalis, l'association est définie comme un: "terme emprunté à l'associationnisme et désignant toute liaison entre deux ou plusieurs éléments psychiques dont la série constitue une chaîne associative» (Laplanche et Pontalis, 2007, p. 36). Pour la signification du mot «libre», je vais simplement retourner à son origine, à son étymologie qui dérive du latin liber, signifiant bel et bien «libre», mais également «sans entrave» ou encore «indépendant». Évidemment, il ne s'agit pas de transposer directement cette méthode analytique à l'enseignement universitaire, mais de garder les fondements auxquels se rattache l'importance de la liberté et de la liaison. Pour sa part, la liberté d'association soulève davantage la possibilité de ne pas s'affilier à un groupe ou à une institution en particulier, donc de demeurer dans cette lignée de l'indépendance. Ces deux conceptions que sont l'association libre et la liberté d'association utilisent somme toute des termes connectés et l'idée de la liberté du sujet ainsi que celle des liens, des associations, seront à garder à l'esprit durant la lecture de la prochaine section. Ensuite, la transmission s'ajoute à la réflexion dans son action même de faire connaître qui s'applique nécessairement au cadre universitaire, mais également dans ce que l'intime de l'expérience de l'analyse a transformé chez un sujet, qui est également un acte de transmission théorique. En bref, l'idée est surtout de pointer l'importance d'une forme de "libération» dans la transmission des différents savoirs, même s'il y a suggestions et transferts dans les différents «enseignements», qu'ils soient universitaires ou «indépendants»: la liberté de penser des liens, dans le partage et la tradition. Je vais tenter de déployer ces idées.

D'abord, ce sera en référence au texte Notre idéologie de formation d'André Lussier, daté du début des années 90 , que des liens pourront se former au sujet de la transmission pour ensuite s'accorder à la lecture de ce glossaire. Dans son article, Lussier élabore autour de la question de la formation dans les instituts psychanalytiques. Pour ma part, je vais tenter de démontrer que sa réflexion spécifique à la formation des analystes peut également s'appliquer 
aux institutions universitaires. Je ne ferai pas un deuxième compte rendu ici, mais j'utiliserai simplement quelques éléments pour alimenter la réflexion sur ce sujet encore d'actualité: où se transmettra la psychanalyse, dans quel lieu, par qui? Pouvons-nous croire qu'il y aura transmission des prémices de l'association libre au-delà des hauts murs universitaires?

Pour sa part, André Lussier affirme qu'un des objectifs de transmission de l'analyste est d'" aider les jeunes générations à devenir ce qu'elles sont». Cela dit, il insistait sur la grande difficulté de ne pas marquer une distinction nette, voire hiérarchique entre, d'une part, le corps enseignant et, d'autre part, celui étudiant. Une telle distinction selon lui empêche grandement la libre association d'avoir cours tout autant que l'ouverture vers une plus grande réciprocité de part et d'autre. Il semblerait que la manière dont André Lussier anticipe la formation à la psychanalyse soit teintée de certaines réserves, notamment en rapport à la notion du savoir. En effet, si nous suivons la logique précédente, nous pouvons peut-être comprendre davantage lorsqu'il formule l'espoir suivant: «Il n'y aurait plus de barricades séparant d'un côté ceux qui savent, et de l'autre ceux qui ne savent pas.» Or, loin de lui l'idée d'aplanir les différences, car il évoque également, en lien avec ce dernier propos, que l'analyste d'expérience a certainement beaucoup à offrir. Néanmoins, il pointe la réflexion vers l'identification mutuelle dans laquelle chacun demeurerait bien conscient de ses propres zones d'ignorance plutôt qu'elles ne se cristallisent dans un transfert de type idéalisant où l'identification pourrait s'assujettir à la pulsion de mort. Ce faisant, où serait donc la transmission qui nécessite de passer par le grand détour de la pulsion de vie, alors que nous connaissons les effets mortifères du transfert idéalisant?

Dans ce glossaire, les analystes se prêtent au jeu du dévoilement, de l'authenticité, sans nier la complexité théorique qui sous-tend ces possibilités cliniques. Ce dévoilement plonge le lecteur dans une possibilité identificatoire plus près de la mutualité et, comme André Lussier le relève, Jacques Mauger aurait dit: "C'est par le biais du silence que l'analyste didacticien devient insurpassable» (cité dans Lussier, 1992, p. 485). À travers ce glossaire, la théorie prend corps, prend voix et nous rappelle la force du partage, donc d'une certaine façon la force de ce que l'on peut transmettre à travers le sensible du savoir psychanalytique. À la fin, André Lussier affirme ceci avec une conviction manifeste dans son article: «il semble que l'on oublie trop souvent le fait que "la psychanalyse ça ne s'enseigne pas"» (Lussier, 1992, p. 487) et que la transmission de la psychanalyse «n'est pas du même ordre 
que la transmission d'un métier à un apprenti». Cette affirmation renvoie à mes questionnements concernant la place - quelle place? - de la psychanalyse à l'université. Comment trouver cette zone d'équilibre, comme l'offre d'une certaine manière le glossaire, entre le savoir théorique et l'être clinique, dans ce que peut soutenir - sous tenir - ce lieu du savoir universitaire?

Pour sa part, Nicolas Lévesque publie Un rêve-manifeste en 2011 où il invite le lecteur à rêver avec lui d'un monde meilleur pour la psychanalyse. Comment nous interpelle-t-il? Il part de sa propre expérience et propose un manifeste où il y aurait un département universitaire de psychanalyse au Québec au sein duquel il y aurait autant une transmission de la théorie qu'une formation clinique non pas d'orientation psychodynamique comme le propose le cursus actuel en psychologie, mais bel et bien analytique. En effet, il écrit ceci: "Quelque chose de la présence de la psychanalyse à l'université avait réussi à se frayer un chemin en moi, malgré tous les vents défavorables. J'étais toutefois né, en tant que psychanalyste, dans un drôle de contexte, une étrange scène primitive qui produit des psychanalystesqui-n'en-sont-pas et des Écoles, des Instituts, vidés de toute relève réelle» (Lévesque, 2011, p. 77). Nous pouvons sentir que pour lui la transmission a, d'une certaine manière, failli à sa mission, considérant la quasi absence de la jeunesse dans les milieux spécifiquement psychanalytiques. Je crois que l'université pourrait être un lieu stable et à même de porter cette mission.

En lien avec ces "psychanalystes-qui-n'en-sont-pas», il écrit qu'il serait dans cette posture «sans affiliation et libre d'association de laquelle pourraient émerger des psychanalystes en devenir, ce qui me fait revenir au texte d'André Lussier lorsqu'il évoque les prémices nécessaires pour être analyste et la règle fondamentale depuis Freud, c'est-à-dire l'association libre. Ces notions de liberté et d'association semblent cruciales pour ces deux hommes; où placer la liberté du sujet, souvent sujet en devenir, dans les transferts multiples soulevés par la posture érigée, irriguée, de nos universités? Peut-être interpellé par la liberté d'association, Nicolas Lévesque soulève, et il est tout à fait important de le souligner, que depuis l'obligation d'effectuer un doctorat afin d'être psychologue, la nécessité de faire une autre formation de type «marathonien » en décourage plus d'un à s'engager dans la formation plus spécifique qu'offrent d'autres institutions psychanalytiques telles que la Société psychanalytique de Montréal (SPM). Or, est-ce que l'université, encore plus de nos jours, peut s'associer au terme qu'est celui de l'indépendance employé par N. Lévesque dans son texte? Comment garder l'esprit analytique (Lussier, 1992) vivant à la lumière des différents 
défis institutionnels qu'imposent les universités, surtout dans leur prise de pouvoir, lieux où le néolibéralisme s'est bien infiltré dans les mailles de la pensée scientifique et éthique. Comment former des analystes dans ce cadre où le savoir apparait souvent colmaté par le pouvoir? Finalement, Nicolas Lévesque évoque un thème qui m’apparait fort pertinent, à savoir celui de la reconnaissance symbolique, avec un «chez-soi», un lieu garant et gardien d'une tradition analytique; d'un savoir qui peut se transmettre et perdurer dans le temps, sous le toit somme toute solide qu'offre l'institution universitaire.

Si l'institution n'a pas de visage, ce sera nécessairement l'incarnation des visages pluriels et, espérons-le, engagés qui porteront l'acte de foi qu'exige la présence des "porteurs-transporteurs» de la psychanalyse au cœur des universités. Pour ma part, il me semble nécessaire d'ouvrir des voies communes où plusieurs voix et lieux de rencontres pourront offrir partage et courage. Spécifions seulement au passage une logique qui, pensons-nous, semble tout à fait évidente: le savoir nécessite d'être partagé, donc transmis, si nous souhaitons garder la même rigueur et la même éthique scientifique proposées par Freud jadis. Or, il faudra également proposer d'autres lieux où l'esprit analytique s'incarnera autrement pour que ce savoir (théorique) se transforme dans la clinique en « un savoir de non-savoir». À ceci, il m’apparait évident que la formation des analystes ne peut s'inscrire au sein de l'institution universitaire, bien que cette dernière puisse certainement offrir une porte, une entrée, dans la réflexion et l'ouverture à ce mode de pensée chez la jeunesse. L'université semble être un lieu tout indiqué pour favoriser certains processus de transmission de la psychanalyse, avec les défis que cela exige. Cette institution pourrait donc jouer son rôle de tiers comme gardien de la tradition où l'on pourrait garder vivants et ancrés les savoirs et les théories diverses, le tout incarné par des analystes aux visages ouverts et curieux. Les institutions analytiques auraient, selon moi, avantage à s'ouvrir, à faire confiance, à rencontrer la jeunesse qui oui, se retrouve beaucoup plus et pour longtemps, dans les universités.

Par ailleurs, un pont s'est tout récemment créé entre la SPM et le Centre d'activités et de références psychodynamique et humaniste (CARPH), et il y aura des midi-conférences offerts par des psychanalystes au cœur du département de psychologie à l'Université du Québec à Montréal. Cela nous renvoie à la question de la demande, du désir. Que me demande l'université? Et l'inverse peut-il advenir; l'étudiant pourra-t-il demander à son tour? Que peut-on demander à un maître si ce n'est que l'espoir d'un pouvoir-savoir? 
La psychanalyse sera-t-elle capable de déjouer ce genre d'impasse (du pouvoir, de la maîtrise, de la vérité) au sein de l'université? Sera-t-elle capable de voir qu'il y a une réciprocité dans la demande, comme le psychanalyste assis seul dans son bureau privé; il y a là une demande, un désir de sa part, mais si personne ne vient cogner à sa porte, restera-t-il là éternellement, pris dans sa tour d'ivoire, de savoir? Enfin, de voir que la psychanalyse se déplace, s'engage, se montre et puisse venir à la rencontre d'autres visages, de jeunes visages. C'est beau. J'ai de l'espoir, je suis cette jeunesse qui souhaite voir se bâtir divers ponts, de préférence en bambou. Parce que le bambou, c'est à la fois souple et solide.

En conclusion, il est bon de lire que la psychanalyse continue à faire son chemin, comme ce rythme mouvementé des grandes marées. De lire que des cliniciens et cliniciennes sont toujours en quête, en devoir - non pas d'une vérité ou d'une technique efficace à exposer - mais bel et bien de réflexions, de partages et d'engagements dans ce que l'intime d'une séance peut transmettre comme savoir. Le savoir analytique ici n'est pas celui d'une théorie qui démontre, mais d'un travail analytique où chacun ayant son style, portant sa propre traversée analytique et ses propres superviseurs dans son historicité éprouvée et marquée. Cela n'enlève en rien la rigueur de créer, toujours avec les autres, à partir de soi. Je vous invite donc à mon tour, comme je l'ai été, à parcourir ce glossaire pour que puisse continuer d'exister la pensée vivante et souvent éclairante de différents cliniciens et cliniciennes analytiques, et pour que puissent se décloisonner les solitudes des bureaux, des institutions, des théories.

Stephany Squires stephany.squires@hotmail.com

\section{Références}

Danon-Boileau, L. et Tamet, J.-Y. (dir.) (2016). Des psychanalystes en séance. Glossaire clinique de psychanalyse contemporaine. Paris: Gallimard.

Laplanche, J. et Pontalis, J.-B. (2007). Vocabulaire de la psychanalyse ( $5^{\mathrm{e}}$ éd.). Paris: Presses universitaires de France.

Lévesque, N. (2011). Un rêve-manifeste. Filigrane, 20 (1), 77-79.

Lussier, A. (1992). Notre idéologie de formation. Revue française de psychanalyse, 56 (2), 483 490. doi:10.3917/rfp.g1992.56n2.0483. 